\title{
Influence of ion beam scattering on the electrical resistivity of platinum hot
}

\section{films}

Masahiro Narasaki, ${ }^{\text {a, b }}$ Haidong Wang, ${ }^{c}$ Yasuyuki Takata, ${ }^{\text {b, c }}$ and Koji Takahashi, ${ }^{a, b, *}$

a Department of Aeronautics and Astronautics, Kyushu University, 744 Motooka, Fukuoka

819-0395, Japan

b International Institute for Carbon-Neutral Energy Research (WPI-I2CNER), Kyushu University, 744 Motooka, Fukuoka 819-0395, Japan

c Department of Mechanical Engineering, Kyushu University, 744 Motooka, Fukuoka 819-0395, Japan

\begin{abstract}
Platinum hot films have been used as precise resistance thermometers to measure the thermal conductivities of carbon nanotubes and graphene. Assisted by focused ion beam (FIB) irradiation, the influence of defects on phonon transport have been examined. However, wide lateral ion beam scattering may affect the electrical properties of hot films and cause uncertainty. In this letter, the effect of FIB irradiation on the electrical resistivity of platinum hot films was evaluated. To investigate this effect qualitatively, electrical resistivity measurement and FIB irradiation were alternated while changing irradiation positions and
\end{abstract}


doses. Irradiated ions were found to travel further than $25 \mu \mathrm{m}$ away from the directly irradiated area, resulting in an increase of electrical resistivity of the film according to total accumulated dose. The number of scattered ions was found to depend on the irradiated surface. An empirical equation describing the relationship between electrical resistivity and assumed ion density in the hot films was proposed. The obtained results enable us to accurately estimate the thermal or electrical properties of nanomaterials using hot-film sensors combined with nanofabrication techniques using FIB.

\section{Keywords}

Platinum nano sensor, Focused ion beam, Electrical resistivity

* Corresponding author. E-mail adress: takahashi@aero.kyushu-u.ac.jp

\section{Introduction}

Platinum $(\mathrm{Pt})$ thin films are widely used to measure the thermal properties of nanomaterials.[1-6] It is well known that calibration of thin films plays an important role in these measurements because the values of their electrical and thermal properties strongly depend on their deposition conditions, geometric size, and defects. In addition, the properties of thin films are quite different from those of the corresponding bulk materials. $[7,8]$ These 
deviations are attributed to the short electron mean free path or phonon mean free path in the thin films caused by surface scattering, $[9,10]$ and grain boundary scattering. $[8,11]$ Recently, Hayashi et al.[12] used a Pt hot-film sensor combined with focused ion beam (FIB) irradiation on carbon nanotubes (CNTs) to unveil ballistic phonon transport. They artificially provoked phonon scattering at the region of the CNT irradiated with the FIB, and examined the dependence of thermal conductivity on the length of the pristine part of the CNT. Wang and co-workers applied this nanomachining technique for the thermal measurement of graphene on a gold hot film.[13] They irradiated graphene with a FIB to make nanoholes and tune its thermal conductivity. These two experimental reports demonstrate that the combination of hot-film sensors with FIB irradiation is a strong tool to experimentally investigate phonon transport in CNTs and graphene in situ. However, the effect of FIB irradiation is known to extend far from the directly irradiated area.[14-16] When a FIB is irradiated near a hot-film sensor, it may change the properties of the hot film. Consequently, it is necessary to understand how the electrical properties of hot films are influenced by FIB irradiation to obtain accurate experimental data.

There are two main reasons why a FIB exerts an influence far from its directly irradiated area.[15,16] One is the Gaussian distribution of a FIB. Liao et al.[15] observed the areas of graphene damaged by a FIB by Raman spectroscopy, and reported that the considerable lateral damage can be attributed to the Gaussian beam shape and unfocused gallium $(\mathrm{Ga})$ ions 
in the FIB. The other reason is residual gas scattering in the vacuum chamber. Thissen and colleagues irradiated a FIB on graphene with residual gas at different background pressures and observed the damaged areas by Raman spectroscopy.[16] They found that the size of the damaged area decreased as pressure was lowered. However, although possible reasons for ion beam scattering have been specified, quantitative evaluation of the effect of a scattered ion beam on hot films has not been performed. In this work, we measured the electrical resistivity of Pt hot films exposed to a scattered Ga ion beam, and proposed an empirical equation that describes the relationship between ion density in the hot film and resistivity.

\section{Experimental}

Measurements were performed in the chamber of a Versa 3D (FEI, USA) equipped with a scanning electron microscope (SEM), FIB, and temperature-controlled stage, as shown in Fig. 1(a). A silicon ( $\mathrm{Si}$ ) substrate with a Pt hot-film sensor was set on the stage. This sensor was fabricated by the MEMS technique, and consisted of two Pt electrodes and heat sinks with a Pt hot film suspended between them, and another Pt heat sink located $5 \mu \mathrm{m}$ away from the hot film. The dimensions of the six Pt hot films used in this study are summarized in Table I. By repeating electrical resistivity measurements of the hot films using the four-probe method just after FIB irradiation scanning parallel to the hot film, we evaluated the effect of FIB irradiation on the electrical resistivity of the hot films. A Ga ion beam was raster scanned 
across an area of $100 \mathrm{~nm} \times 20 \mu \mathrm{m}$ at an accelerating voltage of $30 \mathrm{kV}$. Table II lists the three kinds of FIB conditions used in our experiments. Positions of FIB irradiated area were shifted with respect to the hot film, as illustrated in Fig. 1(b). Temperature and pressure were $300 \mathrm{~K}$ and less than $7 \times 10^{-4} \mathrm{~Pa}$, respectively.

(a)

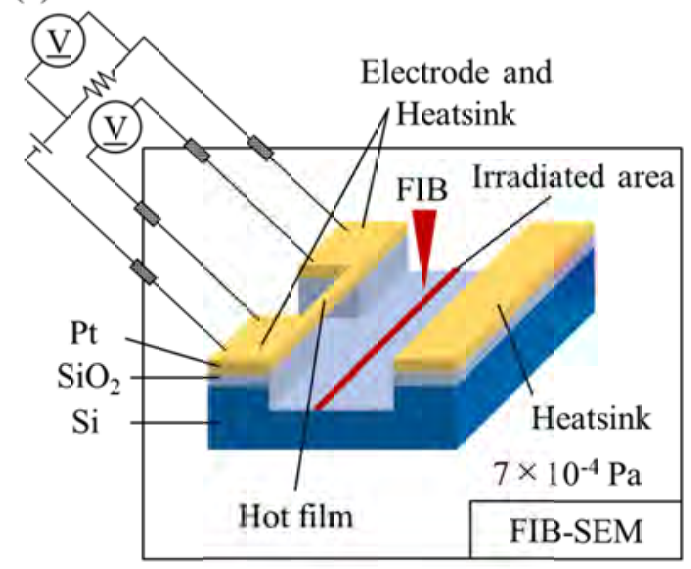

(b)

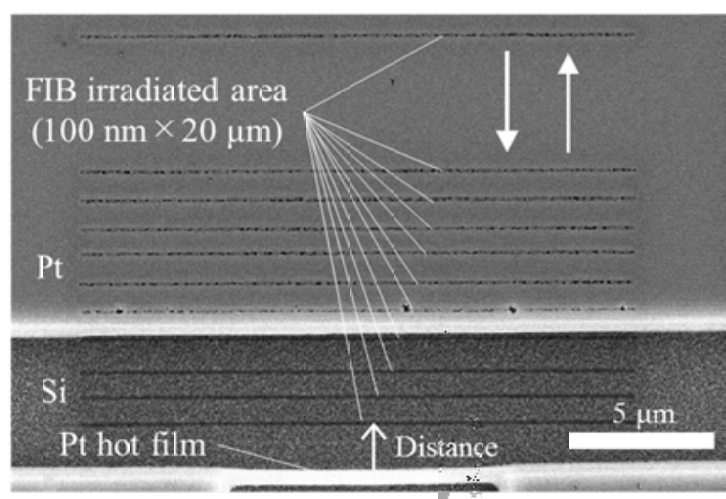

Fig. 1. (a) Schematic of experimental apparatus and FIB irradiation. (b) SEM image of a Pt hot-film sensor after FIB irradiation. The traces of FIB irradiation are observed parallel with the Pt hot film.

TABLE I. Dimensions of Pt hot films.

\begin{tabular}{cccc}
\hline \hline $\begin{array}{c}\text { Pt Hot } \\
\text { film }\end{array}$ & $\begin{array}{c}\text { Length }^{\mathrm{a}} \\
{[\mu \mathrm{m}]}\end{array}$ & $\begin{array}{c}\text { Width }^{\mathrm{a}} \\
{[\mathrm{nm}]}\end{array}$ & $\begin{array}{c}\text { Thickness }^{\mathrm{b}} \\
{[\mathrm{nm}]}\end{array}$ \\
\hline H1 & 9.81 & 418 & 40 \\
H2 & 9.75 & 458 & 40 \\
H3 & 9.70 & 451 & 40 \\
H4 & 9.65 & 549 & 40
\end{tabular}




\begin{tabular}{llll} 
H5 & 9.45 & 536 & 40 \\
H6 & 9.55 & 577 & 40 \\
\hline \hline
\end{tabular}

${ }^{a}$ Length and width were determined by SEM observation.

${ }^{\mathrm{b}} \mathrm{A}$ film thickness meter was used to deposit an arbitrary thickness of Pt.

TABLE II. FIB irradiation conditions.

\begin{tabular}{cccc}
\hline \hline Condition & $\begin{array}{c}\text { Dose } \\
{\left[\mathrm{pC} / \mu \mathrm{m}^{2}\right]}\end{array}$ & $\begin{array}{c}\text { Beam current } \\
{[\mathrm{pA}]}\end{array}$ & $\begin{array}{c}\text { Beam diameter } \\
{[\mathrm{nm}]}\end{array}$ \\
\hline $\mathrm{A}$ & 10.00 & 1.6 & 7.1 \\
$\mathrm{~B}$ & 77.04 & 10.0 & 13.0 \\
$\mathrm{C}$ & 229.84 & 30.0 & 17.0 \\
\hline \hline
\end{tabular}

\section{Results and discussion}

First, the FIB was irradiated at a position $500 \mu \mathrm{m}$ away from the hot films (H1, H2 and H3).

The position of the FIB irradiated area was gradually shifted closer to the hot films up to the shortest distance of $1 \mu \mathrm{m}$. Fig. 2 shows the measured electrical resistivity of the hot films.

The gradual increase of electrical resistivity of these three hot films indicates that scattered ions reached a distance of more than $25 \mu \mathrm{m}$ away from the directly irradiated area. The increase of electrical resistivity of the hot films, $\Delta \rho[\Omega \mathrm{m}]$, is defined as follows.

$$
\Delta \rho=\rho_{i}-\rho_{i-1},
$$

where $\rho_{i}[\Omega \mathrm{m}]$ is the electrical resistivity after ith irradiation, and $\rho_{0}[\Omega \mathrm{m}]$ is the intrinsic electrical resistivity of the film. As indicated in the inset of Fig. 2, a larger dose at a closer 
position results in a larger $\Delta \rho$. The $\Delta \rho$ curves show gaps at $5 \mu \mathrm{m}$ because the irradiated

material changed from $\mathrm{Pt}$ to oxidized $\mathrm{Si}$. Pt atoms are bigger than $\mathrm{Si}$ atoms, so they have a

bigger scattering cross section with the Ga ions in the FIB, resulting in larger $\Delta \rho$.

In the experiment using H4, the position of FIB irradiated area was shifted in the opposite

direction of that using $\mathrm{H} 1, \mathrm{H} 2$ and $\mathrm{H} 3$, starting at a distance of $1 \mu \mathrm{m}$ from the hot film. This is

why the results of $\mathrm{H} 4$ show different tendency from the others in Fig. 2. Comparing H4 and

the others at the shortest distance, $\Delta \rho$ of $\mathrm{H} 4$ is larger than that of $\mathrm{H} 1$ and even $\mathrm{H} 2$ and $\mathrm{H} 3$. At

other distances, however, $\Delta \rho$ of $\mathrm{H} 4$ is smaller and close to zero at a shorter distance than the

others. These results indicate that more Ga ions accumulated by previous irradiations result in smaller $\Delta \rho$ when the same doses are irradiated.

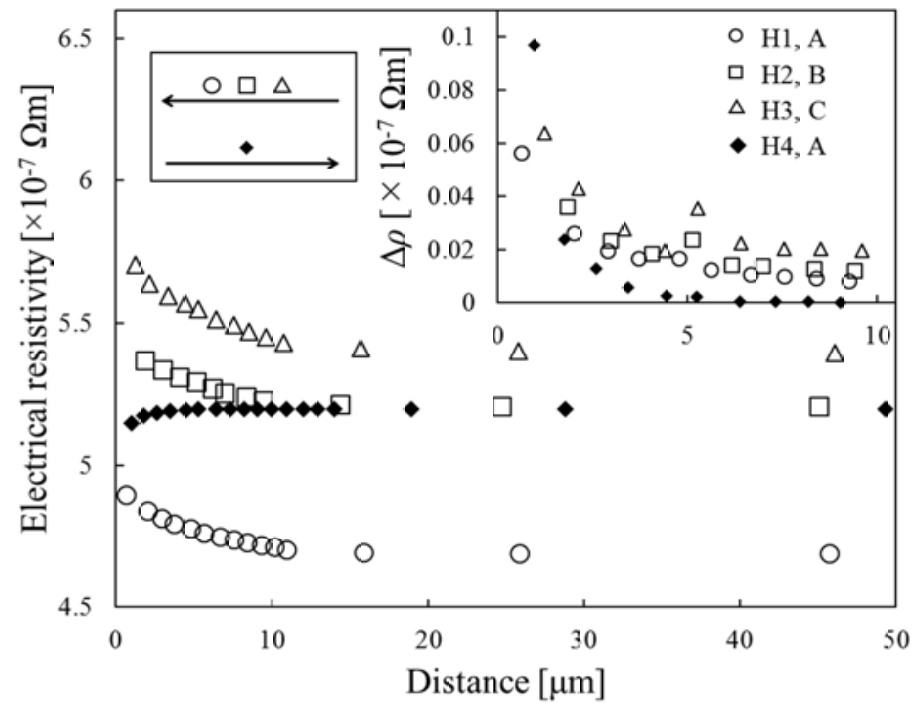

Fig. 2. Measured electrical resistivity of Pt hot films after FIB irradiation at different 
distances from the films. Data for the $\mathrm{H} 1$ hot film under condition A (circles), hot film $\mathrm{H} 2$ under condition B (squares), hot film H3 under condition C (triangles), and hot film $\mathrm{H} 4$ under condition A (diamonds) are shown. The inset shows the increase of resistivity $\Delta \rho$ caused by FIB irradiation at each distance.

To investigate the relationship between electrical resistivity and Ga ion density in the hot films, each hot film was exposed to three different FIB irradiation doses at an identical position $1 \mu \mathrm{m}$ away. The measured resistivity is shown in Fig. 3(a). Irradiated dose was transformed into Ga ion density in the hot films using reported results,[16] which related scattered ion dose with distance from the directly irradiated area. The ion dose $1 \mu \mathrm{m}$ away from the directly irradiated area was $1.5 \times 10^{13}$ ions $/ \mathrm{cm}^{2}$, which was transformed into a Ga ion density in our hot film of $3.8 \times 10^{24}$ ions $/ \mathrm{m}^{3}$. Assuming that the $\mathrm{Ga}$ ion density is proportional to the original ion dose of the FIB irradiation, the relationship between electrical resistivity and estimated density is presented in Fig. 3(b). The dotted curve is the fitting curve expressed as the function of the natural logarithm,

$$
\rho=\rho_{0}+\mathrm{X} \ln (\mathrm{Y} n+1)
$$

where $\rho[\Omega \mathrm{m}]$ is the measured electrical resistivity after FIB irradiation, and $n\left[\right.$ ions $\left./ \mathrm{m}^{3}\right]$ is the Ga ion density in the hot films. $\mathrm{X}[\Omega \mathrm{m}]$ and $\mathrm{Y}\left[\mathrm{m}^{3} /\right.$ ions $]$ determined by the least-squares method were $5.75 \times 10^{-9} \Omega \mathrm{m}$ and $8.53 \times 10^{-25} \mathrm{~m}^{3} /$ ions, respectively. By substituting the total 
accumulated $n$ into equation (2), calculated resistivity was compared with experimental results, as illustrated in Fig. 4. The electrical resistivity obtained from equation (2) is consistent with the experimental results for H4, but not with that for H1. This discrepancy for $\mathrm{H} 1$ is attributed to the smaller intrinsic resistivity of $\mathrm{H} 1\left(4.69 \times 10^{-7} \Omega \mathrm{m}\right)$ than those of $\mathrm{H} 4, \mathrm{H} 5$, and H6, which means that H1 has a bigger grain size and longer electron mean free path compared with those of the other films. The shortening of electron mean free path induced by impurities that leads increased resistivity is larger in the hot films with longer electron mean free path. Indeed, it is worth noting that a proper parameter $X\left(8.65 \times 10^{-9} \Omega \mathrm{m}\right.$ here $)$ enables the calculated results from equation (2) to achieve good consistency with experimental results of $\mathrm{H} 1$, as shown in Fig. 4. As well, equation (2) should work in the case of $\mathrm{H} 2$ and $\mathrm{H} 3$ by estimating $\mathrm{Ga}$ ion density from ref. 16 and by choosing parameters $\mathrm{X}$ and $\mathrm{Y}$ from experimental data.

Lastly, Matthiessen's rule is of some help to discuss the experimental results in Fig. 3. This empirical rule states that the contribution of each electron scattering factor to total electrical resistivity can be dealt with individually. Therefore, the resistivity of a thin film can be expressed as the sum of the resistivities of electron-phonon, surface, grain boundary, and impurity scattering. When an impurity is present as in our case, only the impurity term changes. Generally, the electrical resistivity of bulk metals increases in proportion to their impurity density. However, our results showed a logarithmic resistivity increase. To reveal the 
underlying physics of this discrepancy, further study considering the effects of Ga ions on electron scattering in detail is desired.
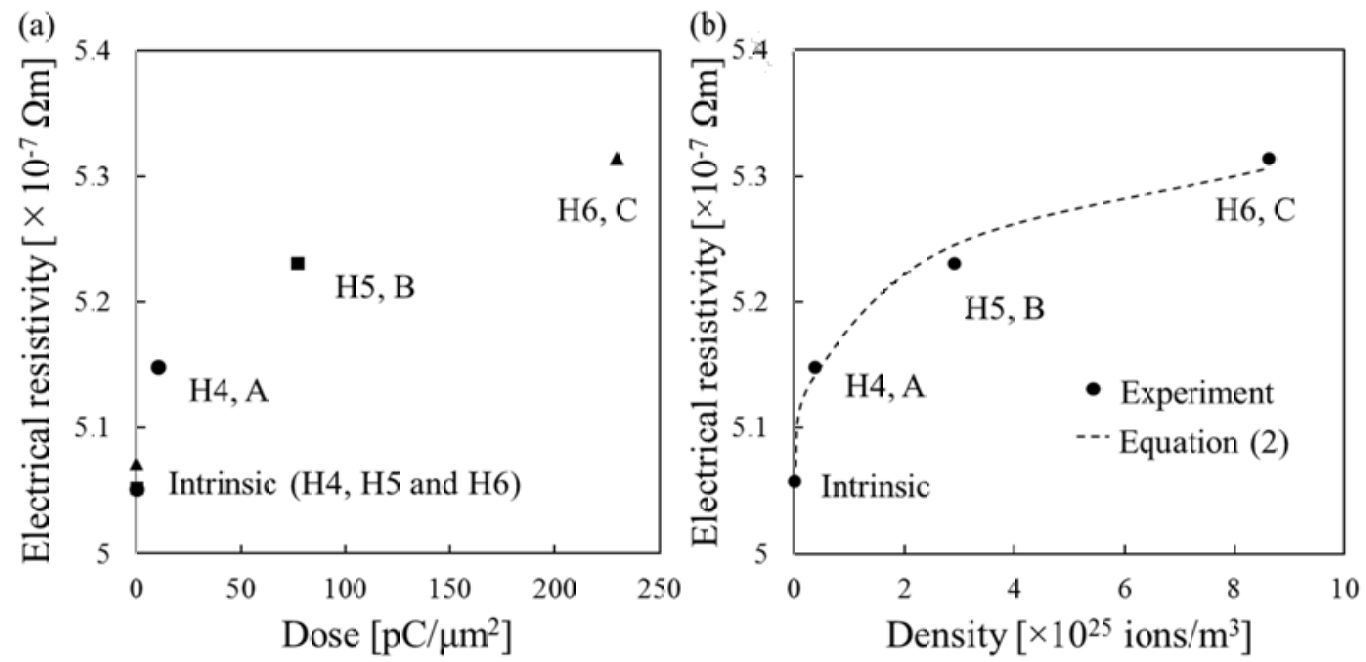

Fig. 3. (a) Measured electrical resistivity of Pt hot films H4, H5 and H6 after FIB irradiation under condition A, B, and C, respectively. The intrinsic resistivity of these films is also listed. (b) Measured electrical resistivity as a function of Ga ion density estimated using reported data.[16] The dotted line is the least-squares fitting curve obtained from equation (2). 


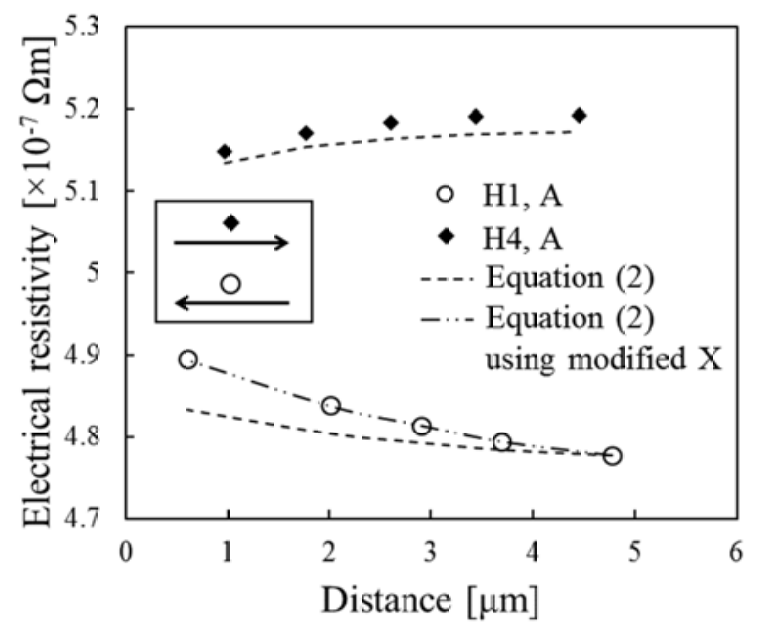

Fig. 4. Measured electrical resistivity of Pt hot films H1 under condition A (circles), and H4 under condition A (solid diamonds). The dotted lines were estimated using equation (2). The dashed-dotted line shows X modified to match with experimental results of H1.

\section{Conclusion}

In conclusion, we measured the electrical resistivity of Pt hot films exposed to FIB irradiation at different distances. The longest distance that scattered ions traveled exceeded 25 $\mu \mathrm{m}$, and ion scattering was slightly affected by the irradiated materials. Furthermore, electrical resistivity depended on the total accumulated Ga ions. We proposed an empirical equation to explain the relationship between electrical resistivity and $\mathrm{Ga}$ ion density in Pt hot films. This equation is useful to facilitate study of nanomaterials using hot-film sensors with FIB irradiation. 


\section{Acknowledgement}

This work was partially supported by CREST, JST, and JSPS KAKENHI Grants 26289047

and 16H04280. M. N. acknowledges an I2CNER super research assistantship.

\section{References}

[1] P. Kim, L. Shi, A. Majumdar, P.L. McEuen, Thermal Transport Measurements of Individual Multiwalled Nanotubes, Phys. Rev. Lett. 87 (2001) 215502. doi:10.1103/PhysRevLett.87.215502.

[2] D. Li, Y. Wu, P. Kim, L. Shi, P. Yang, A. Majumdar, Thermal conductivity of individual silicon nanowires, Appl. Phys. Lett. 83 (2003) 2934. doi:10.1063/1.1616981.

[3] M. Fujii, X. Zhang, H. Xie, H. Ago, K. Takahashi, T. Ikuta, H. Abe, T. Shimizu, Measuring the Thermal Conductivity of a Single Carbon Nanotube, Phys. Rev. Lett. 95 (2005) 065502. doi:10.1103/PhysRevLett.95.065502.

[4] C. Yu, L. Shi, Z. Yao, D. Li, A. Majumdar, Thermal Conductance and Thermopower of an Individual Single-Wall Carbon Nanotube, Nano Lett. 5 (2005) 1842-1846. doi:10.1021/n1051044e.

[5] M.T. Pettes, I. Jo, Z. Yao, L. Shi, Influence of Polymeric Residue on the Thermal Conductivity of Suspended Bilayer Graphene, Nano Lett. 11 (2011) 1195-1200. 
doi:10.1021/nl104156y.

[6] H. Hayashi, T. Ikuta, T. Nishiyama, K. Takahashi, Enhanced anisotropic heat conduction in multi-walled carbon nanotubes, J. Appl. Phys. 113 (2013) 014301. doi:10.1063/1.4772612.

[7] X. Zhang, H. Xie, M. Fujii, H. Ago, K. Takahashi, T. Ikuta, H. Abe, T. Shimizu, Thermal and electrical conductivity of a suspended platinum nanofilm, Appl. Phys. Lett. 86 (2005) 171912. doi:10.1063/1.1921350.

[8] H. Wang, J. Liu, X. Zhang, Z. Guo, K. Takahashi, Experimental study on the influences of grain boundary scattering on the charge and heat transport in gold and platinum nanofilms, Heat Mass Transf. 47 (2011) 893-898. doi:10.1007/s00231-011-0825-5.

[9] K. Fuchs, The conductivity of thin metallic films according to the electron theory of metals, Math. Proc. Cambridge Philos. Soc. 34 (1938) 100.

doi:10.1017/S0305004100019952.

[10] E.H. Sondheimer, The mean free path of electrons in metals, Adv. Phys. 1 (1952) 1-42. doi:10.1080/00018735200101151.

[11] A.F. Mayadas, M. Shatzkes, J.F. Janak, Electrical Resistivity Model for Polycrystalline Films: the Case of Specular Reflection At External Surfaces, Appl. Phys. Lett. 14 (1969) 345. doi:10.1063/1.1652680. 
[12] H. Hayashi, K. Takahashi, T. Ikuta, T. Nishiyama, Y. Takata, X. Zhang, Direct evaluation of ballistic phonon transport in a multi-walled carbon nanotube, Appl. Phys. Lett. 104 (2014) 113112. doi:10.1063/1.4869470.

[13] H. Wang, K. Kurata, T. Fukunaga, H. Takamatsu, X. Zhang, T. Ikuta, K. Takahashi, T. Nishiyama, H. Ago, Y. Takata, In-situ measurement of the heat transport in defectengineered free-standing single-layer graphene, Sci. Rep. 6 (2016) 21823. doi:10.1038/srep21823.

[14] G. Spoldi, S. Beuer, M. Rommel, V. Yanev, A.J. Bauer, H. Ryssel, Experimental observation of FIB induced lateral damage on silicon samples, Microelectron. Eng. 86 (2009) 548-551. doi:10.1016/j.mee.2009.01.003.

[15] Z. Liao, T. Zhang, M. Gall, A. Dianat, R. Rosenkranz, R. Jordan, G. Cuniberti, E. Zschech, Lateral damage in graphene carved by high energy focused gallium ion beams, Appl. Phys. Lett. 107 (2015) 013108. doi:10.1063/1.4926647.

[16] N.F.W. Thissen, R.H.J. Vervuurt, J.J.L. Mulders, J.W. Weber, W.M.M. Kessels, A.A. Bol, The effect of residual gas scattering on Ga ion beam patterning of graphene, Appl. Phys. Lett. 107 (2015) 213101. doi:10.1063/1.4936334. 


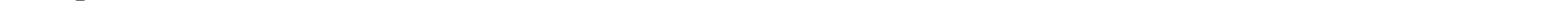

